\title{
FROM CHARACTERISTIC FUNCTION TO DISTRIBUTION FUNCTION: A SIMPLE FRAMEWORK FOR THE THEORY
}

\author{
N.G. SHEPHARD \\ London School of Economics
}

\begin{abstract}
A unified framework is established for the study of the computation of the distribution function from the characteristic function. A new approach to the proof of Gurland's and Gil-Pelaez's univariate inversion theorem is suggested. A multivariate inversion theorem is then derived using this technique.
\end{abstract}

\section{INTRODUCTION}

It is often easier to manipulate characteristic functions than distribution functions. If the characteristic function is known then we can compute the distribution function by using an inversion theorem. This paper reviews the theoretical basis of inverting characteristic functions, presenting the work within a unified framework based on the well-known results of Fourier analysis.

Inverting the characteristic function to find the distribution function has a long history (cf. Lukacs [16, chapter 2]). Lévy's [15] result is the most famous of these theorems, although in this context its practical use is limited to some special cases unless the random variable of interest is always strictly positive (see Bohmann [2] and Knott [14]). Gurland's [10] paper gave a more useful inversion theorem, but it is the paper of Gil-Pelaez [9] which has provided the basis of most of the distributional work completed in this field (cf. Davies [4,5] and Imhof [12]). Gurland's and Gil-Pelaez's results are almost identical. Gurland's is based on the principal value of a Lebesgue integral, while Gil-Pelaez removes the need for principal values by using a Riemann integral. The univariate inversion has been used extensively in econometrics; a short review is given in Phillips [17].

Recently Shively [23] has generalized Gil-Pelaez's work on Riemann integrals to provide a bivariate inversion theorem, while Shively [24] used this expression to tabulate critical values of a statistic proposed by Watson and Engle [25] for testing the stability of the parameters in a regression model. Only Gurland [10] has attempted to provide a multivariate inversion theo-

I thank Dr. M. Knott and Dr. R.W. Farebrother for their comments on earlier versions of this work. The comments of Professor P.C.B. Phillips and the referees were also of considerable assistance. 
rem. Our results are slightly different from those obtained by Gurland. In this paper we develop a framework for the analysis of univariate inversion theorems which offers an easy multivariate generalization. The result, which is given in Theorem 5, is an expression that involves terms that are straightforward to compute.

\section{THE UNIVARIATE INVERSION THEOREM}

Bohmann [1] studied inversion theorems using the results of Fourier analysis. His work, which relies on the properties of convolutions, is in keeping with the discussion of characteristic functions given by Feller [8, chapter XV]. Although the subject matter of this paper is rather different from that considered by Bohmann, our general approach will be consistent with his.

To establish our notation we introduce some definitions. Let $F$ denote the distribution function of interest. Suppose its corresponding density, $f$, is integrable in the Lebesgue sense (written $f \in L$, see for example Rubin [19, chapter 1] for an introduction to Lebesgue integrals) and that its characteristic function is defined as $\varphi(t)=\int_{-\infty}^{\infty} e^{i t x} f(x) d x$. We suppose that $\varphi$ is known and we wish to compute $F$ directly from it. The basic result we will use to perform this calculation is the Fourier inversion theorem.

THEOREM 1 (Fourier inversion theorem). Suppose $g, \varphi \in L$, and

$\varphi(t)=\int_{-\infty}^{\infty} e^{i t x} g(x) d x$

then

$g(x)=\frac{1}{2 \pi} \int_{-\infty}^{\infty} \boldsymbol{e}^{-i t x} \boldsymbol{\varphi}(t) d t$,

everywhere.

Proof. Cf. Hewitt and Stromberg [11, p. 409].

The other result which will be central to our development concerns convolutions.

THEOREM 2. If $g, h \in L, g * h(x)=\int_{-\infty}^{\infty} g(x-y) h(y) d y$, and $g$ and $h$ have Fourier transforms $\phi$ and $\psi$, then the Fourier transform of $g^{*} h(x)$ is $\xi(t)=\phi(t) \psi(t)$.

Proof. Cf. Hewitt and Stromberg [11], Theorem (21.31) and Theorem (21.41).

$g^{*} h$ is called the convolution of $g$ and $h$. These two results will be nearly sufficient to enable us to develop all the results we want in this paper. A simple application of the Fourier inversion theorem gives us the following wellknown result. 
COROLLARY 1. If $f, \varphi \in L$, then

$f(x)=\frac{1}{2 \pi} \int_{-\infty}^{\infty} e^{-i t x} \varphi(t) d t$.

Proof. This follows trivially from Theorem 1 .

Equally, following, for example, Feller, we can convolute $F$ with the uniform distribution on $[-h, h]$ and then use Corollary 1 to produce Lévy's important theorem.

COROLLARY 2 (Lévy [15]). If $f, \varphi \in L$, then

$\frac{F(x+h)-F(x-h)}{2 h}=\frac{1}{2 \pi} \int_{-\infty}^{\infty} \frac{\sin h t}{h t} e^{-i t x} \varphi(t) d t$.

Proof. By Corollary 1, as the left-hand side is a density function.

This corollary has been of use to statisticians working in many fields, as it allows the derivation of an algorithm for the calculation of the distribution function from the characteristic function when the random variable of interest is strictly positive (cf. Bohmann [2, p. 238] and, independently, Knott $[14$, p. 431]). However, as Gil-Pelaez [9] noted, when it is not positive this expression cannot be used for this purpose. As a result many writers have abandoned the idea of using convolutions. Gil-Pelaez employed the notion of a Riemann integral (see the proof in Kendall, Stuart and Ord [13, pp. 120121]), while Gurland used a similar idea, but his proof involved the manipulation of principal values of Lebesgue integrals. Theorem 3 shows that under weak regularity (which will be relaxed to some extent in Theorem 4) this diversion was unnecessary and so allows us to get away from a type of derivation which ". . . detracts from the logical structure of the theory" (Feller [8, p. 511]). The theorem follows by using the Fourier inversion theorem on the convolution of $f$ with a sign function defined on the range $[-h, h]$. As $h \rightarrow \infty$ the Riemann-Lebesgue theorem gives the result.

THEOREM 3. If $f, \varphi \in L$, then under the assumption of the existence of a mean for the random variable of interest

$F(x)=\frac{1}{2}-\frac{1}{2 \pi} \int_{0}^{\infty} \Delta\left[\frac{\varphi(t) e^{-i t x}}{i t}\right] d t$,

where $\underset{t}{\Delta} \eta(t)=\eta(t)+\eta(-t)$.

Proof. Given in the appendix.

The derivative of (3) gives the result in Corollary 1 immediately.

The requirement that $\varphi \in L$ can be removed by using an additional convolution to improve the behavior of the tails of the integrand. In the next theorem we do this by employing Fejér's kernel (there are, of course, many other kernels we could have used, e.g., Abel's or Gauss's). 
THEOREM 4. If $f \in L$, then under the assumption of the existence of a mean

$F(x)=\frac{1}{2}-\frac{1}{2 \pi} \lim _{n \rightarrow \infty} \int_{0}^{n}\left[1-\frac{t}{n}\right] \Delta\left[\frac{\varphi(t) e^{-i t x}}{i t}\right] d t$.

Proof. Given in the appendix.

The existence of the mean is assumed to ensure that $\Delta(\varphi(t) \exp (-i t x) / i t)$ is uniformly bounded. Its existence is a sufficient, although not a necessary condition for this result to hold. It can be removed altogether by using the principal value of the integral-see Gurland [10].

\section{THE MULTIVARIATE INVERSION THEOREM}

Suppose we now become interested in the multivariate generalizations of the above theorems. We suppose $f \in L^{p}$ and that

$\varphi(t)=\int_{-\infty}^{\infty} \cdots \int_{-\infty}^{\infty} e^{i t^{\prime} x} f(x) d x$

where $x=\left(x_{1}, \ldots, x_{p}\right)^{\prime}$ and $t=\left(t_{1}, \ldots, t_{p}\right)^{\prime}$ where $p$ is some positive integer. It is well known that the Fourier inversion theorem and convolution theory go through to the multivariate case, so allowing us trivial proofs of the following well-known corollaries.

COROLLARY 3 (cf. Cramér [3], eqn. 10.6.3). If $f, \varphi \in L^{p}$, then

$f(x)=\frac{1}{(2 \pi)^{p}} \int_{-\infty}^{\infty} \cdots \int_{-\infty}^{\infty} e^{-i t^{\prime} x} \varphi(t) d t$.

Proof. The proof follows using a generalization of the proof of Corollary 1.

Equality (6) is used extensively in the literature - see, for example, Phillips [18] in his work on the sampling behavior of matrix quotients.

COROLLARY 4 (cf. Cramér [3], eqn. 10.6.2). If $f, \varphi \in L^{p}$, and we define an interval $R$ by the inequalities $x_{j}-h<X_{j}<x_{j}+h$ for $j=1, \ldots, p$, then

$\frac{\operatorname{Pr}(R)}{(2 h)^{p}}=\frac{1}{(2 \pi)^{p}} \int_{-\infty}^{\infty} \ldots \int_{-\infty}^{\infty} \prod_{j=1}^{p}\left[\frac{\sin h t_{j}}{h t_{j}}\right] e^{-i t^{\prime} x} \varphi(t) d t$.

Proof. The proof follows using a generalization of the proof of Corollary 2.

Little has been written about the theory of computing the distribution function by inverting multivariate characteristic functions. Gurland [10] extended his univariate procedure to $p$ dimensions using the notion of a prin- 
cipal value of a Lebesgue integral. His result is slightly different from the result we present here. Further, his proof is much more complicated than the one given here. Recently Shively [23,24] has extended Gil-Pelaez's [9] result to two variables in order to calculate some critical values of a test statistic which arises in econometrics. He used Riemann integrals in his derivation and so his proof does not conform with modern work on integrals, as well as being rather obscure.

As we have seen in Section 2 there is no need to use these techniques. The advantage of using a convolution approach is that the multivariate generalizations now follow using standard results.

THEOREM 5. If $f, \varphi \in L^{p}$, then under the assumption of the existence of a mean, the following equality holds.

$\frac{(-2)^{p}}{(2 \pi)^{p}} \int_{0}^{\infty} \cdots \int_{0}^{\infty} \Delta \Delta \cdots t_{t_{1}} t_{t_{2}}\left[\frac{\varphi(t) e^{-i x^{\prime} t}}{i t_{1} i t_{2} \cdots i t_{p}}\right] d t=u^{\dagger}(x)$

where

$$
\begin{aligned}
u^{\dagger}(x)= & 2^{p} F\left(x_{1}, \ldots, x_{p}\right) \\
& -2^{p-1}\left[F\left(x_{2}, x_{3}, \ldots, x_{p}\right)+\cdots+F\left(x_{1}, \ldots, x_{p-2}, x_{p-1}\right)\right] \\
& +2^{p-2}\left[F\left(x_{3}, x_{4}, \ldots, x_{p}\right)+\cdots+F\left(x_{1}, \ldots, x_{p-3}, x_{p-2}\right)\right] \\
& +\cdots+(-1)^{p} .
\end{aligned}
$$

Proof. Given in the appendix.

THEOREM 6. Under the existence of a mean the following equality holds.

$$
\begin{aligned}
\underset{t_{1}}{\Delta \cdots \Delta} t_{t_{p}}\left[\frac{\varphi(t) e^{-i x^{\prime} t}}{i t_{1} i t_{2} \cdots i t_{p}}\right] & =2 i^{p-1}{ }_{t_{2}}^{\Delta} \cdots \Delta_{t_{p}} \operatorname{Im}\left[\frac{\varphi(t) e^{-i x^{\prime} t}}{t_{1} t_{2} \cdots t_{p}}\right], \quad \text { if } p \text { is odd } \\
& =2 i^{p}{ }_{t_{2}} \cdots \Delta_{t_{p}} \operatorname{Re}\left[\frac{\varphi(t) e^{-i x^{\prime} t}}{t_{1} t_{2} \cdots t_{p}}\right], \quad \text { if } p \text { is even. }
\end{aligned}
$$

Proof. Trivial, but extremely tedious manipulations of trigonometric functions.

The assumption of the integrability of $\varphi$ can be removed by following the approach given in the proof of Theorem 4 . This yields a straightforward, although rather ugly, proof of Theorem 7 .

THEOREM 7. If $f \in L^{p}$, then under the assumption of the existence of a mean, the following equality holds.

$$
\frac{(-2)^{p}}{(2 \pi)^{p}} \lim _{n \rightarrow \infty} \int_{0}^{n} \cdots \int_{0}^{n} \prod_{j=1}^{p}\left[1-\frac{t_{j}}{n}\right]{ }_{t_{1}}^{\Delta} t_{t_{2}} \cdots \Delta\left[\frac{\varphi(t) e^{-x^{\prime} t}}{i t_{1} i t_{2} \cdots i t_{p}}\right] d t=u^{\dagger}(x),
$$


where

$$
\begin{aligned}
u^{\dagger}(x)= & 2^{p} F\left(x_{1}, \ldots, x_{p}\right) \\
& -2^{p-1}\left[F\left(x_{2}, x_{3}, \ldots, x_{p}\right)+\cdots+F\left(x_{1}, \ldots, x_{p-2}, x_{p-1}\right)\right] \\
& +2^{p-2}\left[F\left(x_{3}, x_{4}, \ldots, x_{p}\right)+\cdots+F\left(x_{1}, \ldots, x_{p-3}, x_{p-2}\right)\right] \\
& +\cdots+(-1)^{p} .
\end{aligned}
$$

\section{EXAMPLES}

Theorem 3 and the following examples of Theorem 6 may be used to successively generate the distribution functions of $F\left(x_{1}\right), F\left(x_{1}, x_{2}\right), F\left(x_{1}, x_{2}, x_{3}\right)$, and so forth.

$$
\begin{aligned}
& \underline{p=2} \quad \frac{2^{2}}{(2 \pi)^{2}} \int_{0}^{\infty} \int_{0}^{\infty} \Delta_{t_{1}} \Delta\left[\frac{\varphi(t) e^{-i x^{\prime} t}}{i t_{1} i t_{2}}\right] d t_{1} d t_{2} \\
& =\frac{-2^{3}}{(2 \pi)^{2}} \int_{0}^{\infty} \int_{0}^{\infty} \Delta \operatorname{Re}\left[\frac{\varphi(t) e^{-i x^{\prime} t}}{t_{1} t_{2}}\right] d t_{1} d t_{2} \\
& =4 F\left(x_{1}, x_{2}\right)-2\left[F\left(x_{1}\right)+F\left(x_{2}\right)\right]+1 \text {. } \\
& \underline{p=3} \quad \frac{-2^{3}}{(2 \pi)^{3}} \int_{0}^{\infty} \int_{0}^{\infty} \int_{0}^{\infty} \Delta \Delta_{t_{1}} \Delta \Delta\left[\frac{\varphi(t) e^{-i x^{\prime} t}}{i t_{1} i t_{2} i t_{p}}\right] d t_{1} d t_{2} d t_{3} \\
& =\frac{2^{4}}{(2 \pi)^{3}} \int_{0}^{\infty} \int_{0}^{\infty} \int_{0}^{\infty} \Delta t_{t_{2}} \Delta \operatorname{Im}\left[\frac{\varphi(t) e^{-i x^{\prime} t}}{t_{1} t_{2} t_{p}}\right] d t_{1} d t_{2} d t_{3} \\
& =8 F\left(x_{1}, x_{2}, x_{3}\right)-4\left[F\left(x_{1}, x_{2}\right)+F\left(x_{1}, x_{3}\right)+F\left(x_{2}, x_{3}\right)\right] \\
& +2\left[F\left(x_{1}\right)+F\left(x_{2}\right)+F\left(x_{3}\right)\right]-1 \text {. } \\
& \underline{p=4} \quad \frac{2^{4}}{(2 \pi)^{4}} \int_{0}^{\infty} \int_{0}^{\infty} \int_{0}^{\infty} \int_{0}^{\infty} \underset{t_{1}}{\Delta} \underset{t_{2}}{\Delta} \underset{t_{3}}{\Delta} \underset{t_{4}}{\Delta}\left[\frac{\varphi(t) e^{-i x^{\prime} t}}{i t_{1} i t_{2} i t_{3} i t_{4}}\right] d t_{1} d t_{2} d t_{3} d t_{4} \\
& =\frac{2^{5}}{(2 \pi)^{4}} \int_{0}^{\infty} \int_{0}^{\infty} \int_{0}^{\infty} \int_{0}^{\infty} \Delta t_{2} \Delta \Delta \operatorname{se}\left[\frac{\varphi(t) e^{-i x^{\prime} t}}{t_{1} t_{2} t_{3} t_{4}}\right] d t_{1} d t_{2} d t_{3} d t_{4} \\
& =16 F\left(x_{1}, x_{2}, x_{3}, x_{4}\right) \\
& -8\left[F\left(x_{1}, x_{2}, x_{3}\right)+F\left(x_{1}, x_{2}, x_{4}\right)+F\left(x_{1}, x_{3}, x_{4}\right)+F\left(x_{2}, x_{3}, x_{4}\right)\right] \\
& +4\left[F\left(x_{1}, x_{2}\right)+F\left(x_{1}, x_{3}\right)+F\left(x_{1}, x_{4}\right)+F\left(x_{2}, x_{3}\right)\right. \\
& \left.+F\left(x_{2}, x_{4}\right)+F\left(x_{3}, x_{4}\right)\right] \\
& -2\left[F\left(x_{1}\right)+F\left(x_{2}\right)+F\left(x_{3}\right)+F\left(x_{4}\right)\right]+1 \text {. }
\end{aligned}
$$

Numerical integration rules for the evaluation of these integrals are derived in Shephard [20] using Riemann sums with the step sizes automatically cho- 
sen. As such they provide multivariate generalizations of Davies' $[5,6]$ result. The bivariate result is used by Shephard [22] to tabulate Farebrother's [6,7] test for linear restrictions in a heteroskedastic regression model and by Shephard [21] to tabulate the distribution function of the maximum likelihood estimator of a noninvertible moving average process.

\section{CONCLUSION}

This article provides a unified framework for the study of inverting characteristic functions in order to compute the distribution function. The theory is based on the familiar ideas of convolutions, Lebesgue integrals, and Fourier inversions.

The multivariate inversion formula can be applied to a number of important problems in econometrics. The use of Riemann sums to evaluate the integrals allows a straightforward and comparatively cheap method of numerically implementing the theory.

\section{REFERENCES}

1. Bohmann, H. Approximate Fourier analysis of distribution functions. Arkiv für Matematik 4 (1961): 99-1157.

2. Bohmann, H. A method to calculate the distribution function when the characteristic function is known. Nordisk Tidskr. Informationsbehandling (BIT) 10 (1970): 237-242.

3. Cramér, H. Mathematical methods of statistics. Princeton: Princeton University Press, 1946.

4. Davies, R.B. Numerical inversion of a characteristic function. Biometrika 60 (1973): 415-417.

5. Davies, R.B. AS 155: The distribution of a linear combination of $\chi^{2}$ random variables. $A p$ plied Statistics 29 (1980): 323-333.

6. Farebrother, R.W. Testing linear restrictions with unequal variances, a problem. Econometric Theory 4 (1988): 349.

7. Farebrother, R.W. Testing linear restrictions with unequal variances, a solution. Econometric Theory 5 (1989): 324-326.

8. Feller, W. Introduction to Probability Theory and Its Applications, Volume Two. 2nd ed. New York: Wiley, 1971.

9. Gil-Pelaez, J. Note on the inversion theorem. Biometrika 37 (1951): 481-482.

10. Gurland, J. Inversion formulae for the distribution of ratios. Annals of Mathematical Statistics 19 (1948): 228-237.

11. Hewitt, E. \& K.R. Stromberg. Real and Abstract Analysis. New York: Springer-Verlag, 1965.

12. Imhof, J.P. Computing the distribution of quadratic forms in normal variables. Biometrika 48 (1961): 419-426.

13. Kendall, M.G., A. Stuart \& J.K. Ord. Advanced Theory of Statistics, Vol. 1. 5th ed. London: Griffin, 1987.

14. Knott, M. The distribution of the Cramér-von Mises statistic for small sample sizes. Journal of the Royal Statistical Society, Series B 36 (1974): 430-438.

15. Lévy, P. Calcul des probabilités. Paris: Gauthier-Villars, 1925.

16. Lukacs, E. Characteristic Functions. London: Griffin, 1970.

17. Phillips, P.C.B. Exact small sample theory in the simultaneous equations model. In Handbook of Econometrics, Volume 1, Z. Griliches \& M.D. Intriligator (eds.), Amsterdam; North-Holland Publishing Company, 1983. 
18. Phillips, P.C.B. The distribution of matrix quotients. Journal of Multivariate Analysis 16 (1985): $157-161$

19. Rubin, W. Real and Complex Analysis. New York: McGraw-Hill, 1970.

20. Shephard, N.G. Numerical integration rules for multivariate inversions. Journal of Statistical Computation and Simulation 39 (1991): 37-46.

21. Shephard, N.G. Evaluating the distribution function of the maximum likelihood estimator of a first order moving average process and a local level model. Working paper, London School of Economics, 1990.

22. Shephard, N.G. Tabulation of Farebrother's test for linear restrictions in linear regression models under heteroscedasticity. Working paper, London School of Economics, 1990.

23. Shively, T.S. Numerical inversion of a bivariate characteristic function. Working paper, University of Texas at Austin, 1988.

24. Shively, T.S. An analysis of tests for regression coefficient stability. Journal of Econometrics 39 (1988): 367-386.

25. Watson, M.W. \& R.F. Engle. Testing for regression coefficient stability with a stationary AR(1) alternative. Review of Economics and Statistics 67 (1985): 341-346.

\section{APPENDIX}

Proof of Theorem 3. The function $g(y)=\operatorname{sign}(y), y \in[-h, h]$, has the transform

$\varphi_{h}(t)=\int_{-h}^{h} e^{i t y} \operatorname{sign}(y) d y=\frac{2(\cos h t-1)}{i t}$,

which is bounded for all $t$.

The convolution, written $u_{h}(x)$, of $g(y)$ with the continuous density $f(x)$ is $2 F(x)-F(x+h)-F(x-h)$ which, although bounded, is not integrable as $h \rightarrow \infty$. The convolution has the transform $2 \varphi(t)(\cos h t-1) / i t$, which is integrable as $\varphi \in L$. Hence, for fixed $h$ we can use the inversion theorem to give the equality

$$
\begin{aligned}
& \frac{2}{2 \pi} \int_{-\infty}^{\infty} \frac{(\cos h t-1)}{i t} \varphi(t) e^{-i x t} d t, \\
& \quad=\frac{2}{2 \pi} \int_{0}^{\infty}(\cos h t-1) \Delta\left[\frac{\varphi(t) e^{-i x t}}{i t}\right] d t=u_{h}(x) .
\end{aligned}
$$

As $h$ increases, cos $h t$ moves increasing rapidly and so the part of the integrand which includes cos $h t$ makes a diminishing contribution to the integral. A sufficient condition to uniformly bound $\Delta(\varphi(t) \exp (-i x t) / i t)$ is that $\varphi(t)$ is differentiable near zero, which will be true if the mean exists. The consequence of this bounding is that the integral of this function will exist. As a result, in the limit as $h \rightarrow \infty$ the left-hand side of the (A2) can be reduced to, using the Riemann-Lebesgue theorem (cf. Feller [8, p. 513])

$$
\frac{-2}{2 \pi} \int_{0}^{\infty} \Delta\left[\frac{\varphi(t) e^{-i t x}}{i t}\right] d t=2 F(x)-1 \text {. }
$$


Proof of Theorem 4. Recall the proof of Theorem 3. To improve the behavior of the tails of $u_{h}(x)$ we convolute it with Fejér's kernel

$k_{n}(x)=\frac{1}{2 \pi} n\left[\frac{\sin (n x / 2)}{(n x / 2)}\right]^{2}$.

It is important to note that

(i) $\int_{-\infty}^{\infty} k_{n}(x) d x=1$.

(ii) The transform of Fejér's kernel is

$$
\varphi_{n}(t)=\left[1-\frac{|t|}{n}\right] I(|t|<n),
$$

where $I(\cdot)$ is an indicator function.

For fixed $n$ the convolution of $u_{h}(x)$ with $k_{n}(x)$, and its transform, are integrable, as $\varphi(t)$ is bounded. Hence we can use the inversion theorem to give the equality

$$
\begin{aligned}
& \frac{2}{2 \pi} \int_{-\infty}^{\infty}\left[1-\frac{|t|}{n}\right] I(|t|<n)(\cos h t-1)\left[\frac{\varphi(t) e^{-i x t}}{i t}\right] d t \\
& \quad=\frac{2}{2 \pi} \int_{0}^{\infty}\left[1-\frac{|t|}{n}\right] I(t<n)(\cos h t-1) \Delta\left[\frac{\varphi(t) e^{-i x t}}{i t}\right] d t \\
& =u_{h} * k_{n}(x) .
\end{aligned}
$$

When $h \rightarrow \infty$ the left-hand side of (A.6) can be manipulated using the RiemannLebesgue theorem (cf. Feller [8, p. 513]) because for fixed $n$

$\left[1-\frac{|t|}{n}\right] I(t<n) \Delta\left[\frac{\varphi(t) e^{-i x t}}{i t}\right]$

is integrable, as $\Delta(\varphi(t) \exp (-i x t) / i t)$ is bounded due to the assumption of the existence of a mean for the density $f(x)$-see the proof of Theorem 3. Hence the lefthand side of (A.6) reduces to

$\frac{-2}{2 \pi} \int_{0}^{\infty}\left[1-\frac{t}{n}\right] I(t<n) \Delta\left[\frac{\varphi(t) e^{-i x t}}{i t}\right] d t$.

Now think of the right-hand side of (A6) as $h \rightarrow \infty$. Remember it is

$\int_{-\infty}^{\infty}[2 F(x-y)-F(x-y-h)-F(x-y+h)] k_{n}(y) d y$.

As $2 F(x-y)-F(x-y-h)-F(x-y+h)$ is uniformly bounded and $k_{n}(y)$ is integrable (see (A.4)) we can employ the Lebesgue-dominated convergence theorem (see Hewitt and Stromberg [11, Section 12.34]) to imply

$\lim _{h \rightarrow \infty} u_{h} * k_{n}(x)=u^{\dagger} * k_{n}(x)$, 
where $u^{\dagger}(x)=2 F(x)-1$. Thus,

$\frac{-2}{2 \pi} \int_{0}^{\infty}\left[1-\frac{t}{n}\right] I(t<n)\left[\frac{\varphi(t) e^{-i x t}}{i t}\right] d t=u^{\dagger} * k_{n}(x)$.

We now become interested in the behavior of this equality as $n \rightarrow \infty$. Proposition 1 deals with the right-hand side. This is a well-known theory (cf. Hewitt and Stromberg [11, Section 21.42]) although we are using slightly different assumptions from those usually employed and so we include its proof in this appendix. Hence

$\frac{-2}{2 \pi} \lim _{n \rightarrow \infty} \int_{0}^{n}\left[1-\frac{t}{n}\right]{ }_{t}\left[\frac{\varphi(t) e^{-i x t}}{i t}\right] d t=2 F(x)-1$.

Proof of Theorem 5. Define

$g\left(y_{1}, \ldots, y_{p}\right)=\prod_{j=1}^{p} \operatorname{sign}\left(y_{j}\right), \quad$ where $\quad y_{j} \in[-h, h]$,

so it has the transform

$\varphi_{h}(t)=\prod_{j=1}^{p} \frac{2\left(\cos t_{j} h-1\right)}{i t_{j}}$

Consider the convolution of the density function, $f$, with $g\left(y_{1}, \ldots, y_{p}\right)$, which we will write as $u_{h}$

$$
\begin{aligned}
u_{h}(x) & =\int_{-h}^{h} \cdots \int_{-h}^{h} f\left(x_{1}-y_{1}, \ldots, x_{p}-y_{p}\right) g(y) d y, \\
& =\sum_{a_{1}, a_{2}, \ldots, a_{p}} \psi_{h a_{1}, h a_{2}, \ldots, h a_{p}},
\end{aligned}
$$

where the summation is taken over all the values $\left(a_{j}= \pm 1\right)$, with

$\psi_{h_{1}, h_{2}, \ldots, h_{p}}=\sum_{b_{1}, \ldots, b_{D}}(-1)^{b_{1}+\cdots+b_{p}} F\left(x_{1}-h_{1} b_{1}, \ldots, x_{p}-h_{p} b_{p}\right)$,

where the summation is taken over the binary numbers $\left(b_{j}=0,1\right)$ and $F$ denotes a generic distribution function. By the (multivariate) Fourier inversion theorem,

$$
\begin{aligned}
& \frac{2^{p}}{(2 \pi)^{p}} \int_{-\infty}^{\infty} \cdots \int_{-\infty}^{\infty} \varphi(t) e^{-i x^{\prime} t} \prod_{j=1}^{p}\left[\frac{\cos t_{j} h-1}{i t_{j}}\right] d t, \\
& =\frac{2^{p}}{(2 \pi)^{p}} \int_{0}^{\infty} \cdots \int_{0}^{\infty}{ }_{t_{1}} \Delta t_{2} \cdots \Delta t_{t_{p}}\left[\frac{\varphi(t) e^{-i x^{\prime} t}}{i t_{1} i t_{2} \cdots i t_{p}}\right] \prod_{j=1}^{p}\left(\cos t_{j} h-1\right) d t=u_{h}(x) .
\end{aligned}
$$

Allowing $h \rightarrow \infty$, the left-hand side can be simplified by exploiting the RiemannLebesgue theorem. The result is that we have

$\frac{(-2)^{p}}{(2 \pi)^{p}} \int_{0}^{\infty} \cdots \int_{0}^{\infty} \Delta \frac{\Delta}{t_{1}} t_{2} \cdots \Delta\left[\frac{\varphi(t) e^{-i x^{\prime} t}}{i t_{1} i t_{2} \cdots i t_{p}}\right] d t=u^{\dagger}(x)$. 


\section{PROPOSITION 1.}

$\lim _{n \rightarrow \infty} u^{\dagger} * k_{n}(x)=u^{\dagger}(x)$.

Proof.

$$
\begin{aligned}
u^{\dagger} * k_{n}(x)-u^{\dagger}(x) & =\int_{-\infty}^{\infty}\left(u^{\dagger}(x-t)-u^{\dagger}(x)\right) k_{n}(t) d t \\
& =\int_{0}^{\infty} \beta(t) k_{n}(t) d t,
\end{aligned}
$$

where $\beta(t)=u^{\dagger}(x+t)+u^{\dagger}(x-t)-2 u^{\dagger}(x)$. As $u^{\dagger}(x)=2 F(x)-1, \beta(t)$ cannot be integrable for its tails do not go to zero as $|t| \rightarrow \infty$, even though it is uniformly bounded. Choose $\epsilon_{1}>0$, then using the continuity of the distribution function we can choose $\epsilon>0$ so small that $|\beta(t)|<\epsilon_{1} \forall t \in[0, \epsilon)$. Since $k_{n}(t) \leq 2 / \pi n t^{2}$, we have

$$
\begin{aligned}
\left|\int_{0}^{\infty} k_{n}(t) \beta(t) d t\right| & \leq\left|\int_{0}^{\epsilon} k_{n}(t) \beta(t) d t\right|+\left|\int_{\epsilon}^{\infty} k_{n}(t) \beta(t) d t\right| \\
& \leq \epsilon_{1}+\sup _{t}|\beta(t)|\left|\int_{\epsilon}^{\infty} k_{n}(t) d t\right| \\
& \leq \epsilon_{1}+\frac{2}{\pi n} \sup _{t}|\beta(t)| \int_{\epsilon}^{\infty} \frac{1}{t^{2}} d t \\
& \leq \epsilon_{1}+\frac{2}{\epsilon \pi n} \sup _{t}|\beta(t)|,
\end{aligned}
$$

so for $n>2 \sup |\beta(t)| / \epsilon \epsilon_{1} \pi$ we have the required result by choosing $\epsilon_{1}$ sufficiently small. 